\title{
Trial-by-Trial Coupling of Concurrent Electroencephalogram and Functional Magnetic Resonance Imaging Identifies the Dynamics of Performance Monitoring
}

\author{
Stefan Debener, ${ }^{1,2 *}$ Markus Ullsperger, ${ }^{3 *}$ Markus Siegel, ${ }^{1}$ Katja Fiehler, ${ }^{3,4}$ D. Yves von Cramon, ${ }^{3}$ and Andreas K. Engel ${ }^{1}$ \\ ${ }^{1}$ Institute of Neurophysiology and Pathophysiology, Center of Experimental Medicine, University Medical Center, Hamburg University, D-20246 Hamburg, \\ Germany, ${ }^{2}$ Medical Research Council Institute of Hearing Research Southampton, Royal South Hants Hospital, SO 14 OYG Southampton Hants, United \\ Kingdom, ${ }^{3}$ Department of Cognitive Neurology, Max-Planck Institute for Human Cognitive and Brain Sciences, D-04103 Leipzig, Germany, and ${ }^{4}$ Cognitive \\ Psychophysiology Laboratory, Department of Psychology, Philipps-University Marburg, D-35032 Marburg, Germany
}

Goal-directed behavior requires the continuous monitoring and dynamic adjustment of ongoing actions. Here, we report a direct coupling between the event-related electroencephalogram (EEG), functional magnetic resonance imaging (fMRI), and behavioral measures of performance monitoring in humans. By applying independent component analysis to EEG signals recorded simultaneously with fMRI, we found the single-trial error-related negativity of the EEG to be systematically related to behavior in the subsequent trial, thereby reflecting immediate behavioral adjustments of a cognitive performance monitoring system. Moreover, this trial-by-trial EEG measure of performance monitoring predicted the fMRI activity in the rostral cingulate zone, a brain region thought to play a key role in processing of response errors. We conclude that investigations of the dynamic coupling between EEG and fMRI provide a powerful approach for the study of higher order brain functions.

Key words: fMRI; EEG; anterior cingulate cortex; performance monitoring; ICA; error processing

\section{Introduction}

In a rapidly changing environment, goal-directed behavior requires the monitoring and dynamic adjustment of ongoing actions. Erroneous actions, in particular, are highly informative for successful adjustments of future behavior (Ridderinkhof et al., 2004). Accordingly, neural correlates of performance monitoring have been studied intensively in humans by means of electroencephalogram (EEG) and functional magnetic resonance (MR) imaging (fMRI). One of the EEG signatures is the error-related negativity (ERN), an event-related brain potential (ERP) peaking within $100 \mathrm{~ms}$ after erroneous responses (Falkenstein et al., 1990; Gehring et al., 1993). fMRI studies consistently implicate the posterior frontomedian cortex in processing of response errors, negative feedback, response conflict, and decision uncertainty (Ridderinkhof et al., 2004). Informed by both ERN and fMRI research, the current view is that error processing is accomplished by a fundamental performance monitoring system signaling the need for behavioral adjustments in the service of action outcome optimization (Ridderinkhof et al., 2004; Ullsperger and von Cramon, 2004).

Received Aug. 5, 2005; revised Sept. 30, 2005; accepted Nov. 6, 2005

This work was supported by a grant from the Deutsche Forschungsgemeinschaft to M.U. and K.F. We thank R. Niazy, S. Makeig, and A. Delorme for invaluable contributions to this project and S. Zysset and T. Mildner for their help with data recordings.

*S.D. and M.U. contributed equally to this work.

Correspondence should be addressed to Dr. Stefan Debener, Medical Research Council Institute of Hearing Research Southampton, Royal South Hants Hospital, S0 14 0YG Southampton Hants, UK. E-mail: stefan@debener.de. DOI:10.1523/JNEUROSCI.3286-05.2005

Copyright $\odot 2005$ Society for Neuroscience $\quad 0270-6474 / 05 / 2511730-08 \$ 15.00 / 0$
A common notion is that performance monitoring is a dynamic process that systematically fluctuates over time. Behavioral adjustments can, for instance, lead to prolonged reaction times (RTs) on trials subsequent to errors, thereby reflecting a more cautious response mode (Rabbitt, 1966; Ridderinkhof et al., 2004). This view is supported by fMRI work showing that enhanced activity in the posterior frontomedian cortex predicts greater subsequent posterror slowing (Garavan et al., 2002; Kerns et al., 2004). For the ERN, this question is difficult to address, because ERPs usually are derived by averaging across trials. To account for this problem, independent component (IC) analysis (ICA) can be applied, a statistical source separation technique suitable for single-trial EEG analysis (Makeig et al., 2002, 2004). If the ERN indeed reflects performance monitoring, ICA-filtered trial-to-trial variations of its amplitude should go along with systematic behavioral changes in the same trial and particularly in subsequent trials. Based on a previous account (Gehring et al., 1993), we predicted greater single-trial ERN amplitudes after errors, reflecting a change to a more conservative response strategy in subsequent trials.

EEG source localization studies (Dehaene et al., 1994; Ullsperger and von Cramon, 2001) have suggested the posterior frontomedian cortex as neural generator of the ERN. However, these analyses do not inform on whether the ERN is related to hemodynamic changes of error monitoring. To date, it remains poorly understood how hemodynamic and electrophysiological correlates of cognitive processes relate to each other. Regarding the relationship between electrophysiological measures and 
fMRI, local field potentials recorded in the visual cortex of anesthetized monkeys have been shown to predict the local fMRI blood-oxygen-level-dependent (BOLD) signal (Logothetis et al., 2001). Here, we tested whether the electrophysiological correlate of performance monitoring systematically predicts the BOLD response in the rostral cingulate zone (RCZ) of the posterior frontomedian cortex. To address both the dynamic variability of performance monitoring and the relationship between its hemodynamic and electrical signatures, we performed a singletrial analysis of simultaneous EEG/fMRI measurements. We thereby avoided the usual problem that within-subject behavior fluctuates across separate recording sessions (Ullsperger and von Cramon, 2001).

\section{Materials and Methods}

Participants. Eighteen healthy right-handed volunteers participated in the experiment. As a result of technical malfunction and thus incomplete recordings of either EEG or fMRI, data from five subjects had to be discarded. The final sample consisted of eight females and five males (22-29 years of age; mean age, 25.2 years). Written informed consent before the start of the experiment was obtained from each participant according to the declaration of Helsinki.

Behavioral task. Stimuli were presented using Presentation 0.76 (Neurobehavioral Systems, San Francisco, CA) and appeared on a backprojection screen mounted inside the scanner bore behind the participants head. A speeded modified flanker task was used known to yield sufficient error rates to study the ERN (see Fig. 1). Participants were presented with a fixation mark at the center of the screen, after which four horizontal flanker arrows appeared for $110 \mathrm{~ms}$. The arrows were $0.46^{\circ}$ tall and $1.08^{\circ}$ wide and appeared $0.52^{\circ}$ and $1.04^{\circ}$ above and below the screen center. The target arrow was presented for $30 \mathrm{~ms}$ in the center of the flanker arrows; its onset was delayed by $80 \mathrm{~ms}$ from the onset of the flanker. In $50 \%$ of the 400 trials, the flankers pointed in the same direction as the target (compatible trials), and in the other half of the trials in the opposite direction (incompatible trials). Compatible and incompatible trials appeared in randomized order. Participants were instructed to respond with maximal speed and accuracy to the target arrow with the response hand indicated by the arrow direction. Whenever participants responded after an individual, dynamically adapting response deadline, a symbolic feedback was presented for $1400 \mathrm{~ms}$ after target onset, instructing participants to speed up. The average intertrial interval amounted to $6 \mathrm{~s}$; for the remaining time, the fixation mark was presented. The trials were interspersed with a total of 32 nonevents, during which only the fixation cross was presented and no response was required. Trials occurred at multiple, systematically offset time points (range, $0-1.5 \mathrm{~s}$ ) in relation to fMRI data acquisition to improve temporal resolution (Josephs et al., 1997; Miezin et al., 2000).

Simultaneous EEG/fMRI recording. Imaging was performed at 3 tesla on a Siemens (Erlangen, Germany) Trio system equipped with the standard bird cage head coil. Twenty-two functional slices were obtained parallel to the anterior commissure-posterior commissure line (thickness, $4 \mathrm{~mm}$; interslice gap, $1 \mathrm{~mm}$ ) using a gradient-echo echo planar imaging (EPI) sequence with an echo time of $30 \mathrm{~ms}$, a flip angle of $90^{\circ}$, a repetition time (TR) of $2000 \mathrm{~ms}$, and an acquisition bandwidth of 100 $\mathrm{kHz}$. Acquisition of the slices was arranged such that they all were acquired within $1500 \mathrm{~ms}$ and were followed by a $500 \mathrm{~ms}$ no-acquisition period to complete the TR. This was done to visually monitor proper recording of the EEG signal during MR scanning and to include for each TR a nongradient contaminated baseline period into the EEG recordings (Fig. S1, available at www.jneurosci.org as supplemental material). The fMRI matrix acquired was $64 \times 64$ with a field of view of $19.2 \mathrm{~cm}$, resulting in an in-plane resolution of $3 \times 3 \mathrm{~mm}^{2}$. A total of 1309 volumes was acquired. Functional data were motion-corrected off-line with the Siemens motion correction protocol. Before the functional runs, anatomical modified driven equilibrium Fourier transform (MDEFT) and EPI-T1 slices in the plane with functional images were collected.

Continuous EEG data were collected from 30 standard scalp sites using the BrainAmps MR plus, a high-input impedance amplifier specifically designed for recordings in high magnetic fields (BrainProducts, Munich, Germany). Sintered $\mathrm{Ag} / \mathrm{AgCl}$ ring electrodes with built-in $5 \mathrm{k} \Omega$ resistors were used and mounted into an electrode cap according to the 10-20 system (Falk Minow Services, Herrsching, Germany). Two additional electrodes were placed below the left eye and on the lower back to monitor eyeblinks and electrocardiograms, respectively. Electrode impedances were maintained below $10 \mathrm{k} \Omega$ before recordings. The nonmagnetic EEG amplifier was fixed beside the head coil and powered by a rechargeable power pack placed outside the scanner bore. The subject's head was immobilized using vacuum cushions and sponge pads. The amplified EEG signals were transmitted with a fiber optic cable to a recording personal computer placed outside the scanner room. All 32 channels were recorded with $\mathrm{FCz}$ as reference. Although this is an unusual reference site for ERN studies, it allowed us to keep the distance between recording reference and "active" electrodes small, thereby minimizing the chance of amplifier saturation. The data were recorded with a passband of $0.016-250 \mathrm{~Hz}$ and digitized with 5000 samples per second at 16 bit with $0.5 \mu \mathrm{V}$ resolution (dynamic range, $16.38 \mathrm{mV}$ ).

EEG data analysis. EEG data were corrected for MR gradient and ballistocardiac artifacts by applying modified versions of the algorithms proposed by Allen and colleagues (Allen et al., 1998, 2000). Gradient artifacts were removed as implemented in Vision Analyzer 1.04 software (BrainProducts) by subtracting an artifact template from the $40 \mathrm{~Hz}$ lowpass-filtered data, using a baseline-corrected sliding average of 20 consecutive volumes. This resulted in EEGs denoised for MR gradients, as shown for representative $10 \mathrm{~s}$ traces in Figure S1 (available at www. jneurosci.org as supplemental material). Further processing of the 250 $\mathrm{Hz}$ downsampled data was performed using Matlab 6.5 (MathWorks, Natick, MA) and EEGLAB 4.51 (Delorme and Makeig, 2004), a freely available open source software toolbox (EEGLAB toolbox for single-trial EEG data analysis, Swartz Center for Computational Neurosciences, La Jolla, CA; http://www.sccn.ucsd.edu/eeglab). The EEGLAB plug-in FMRIB 1.0 (Niazy et al., 2005) (FMRIB EEGLAB plug-in for removal of fMRI-related artifacts, Center for functional MRI of the Brain, Oxford, UK; http://www.fmrib.ox.ac.uk/ rami/fmribplugin) was used for $0.4-35 \mathrm{~Hz}$ filtered data to remove ballistocardiac artifacts. Based on the identified heartbeat events, an artifact template was defined as the median across a sliding window of 30 heartbeats centered around the heartbeat event being processed. As a result, EEG data denoised for ballistocardiac artifacts were derived but with common EEG artifacts such as eyeblinks still being present (Fig. S1, available at www.jneurosci.org as supplemental material).

The MR-denoised EEG data were re-referenced to common average, and stimulus- and response-locked ERPs were calculated separately for the experimental conditions of interest. The time-locking event for all stimulus-locked analyses was the target arrow onset, with a baseline set to $-200-0 \mathrm{~ms}$. For response-locked ERPs, we used a baseline from -600 to $-400 \mathrm{~ms}$ to avoid contamination of the baseline period with stimulusevoked potentials. Stimulus-locked ERPs clearly indicated the common ERP morphology (i.e., an N1 at occipital channels, a P300 at parietal channels, and the ERN at frontocentral channels), altogether confirming reasonable data quality (Fig. S2a, available at www.jneurosci.org as supplemental material). Grand mean ERP images were computed by colorcoding the single-trial amplitudes aligned to stimulus onset, smoothed with a moving average across 30 adjacent trials (Delorme and Makeig, 2004). Topographical inspection of both scalp ERPs and reaction-timesorted ERP images clearly indicated a contamination of the event-related portion of the signal with different artifacts (Fig. S2, available at www. jneurosci.org as supplemental material). In addition to the typical eyeblink artifacts, a strong response-locked exogenous artifact was visible at temporal sites, as characterized by a reversed polarity between left and right hemisphere channels. This event-related artifact probably was caused by button-press-related small body movements and related current induction. For this and other reasons, it was inevitable to linearly decompose the response-related process of interest from these and further signal contributions. We performed extended infomax ICA (Bell and Sejnowski, 1995; Lee et al., 1999) on the MR-denoised single-subject continuous EEG data. ICA finds an unmixing square matrix of the size of the number of channels, which, when matrix-multiplied with the raw 
data, reveals maximally temporally independent activations. A weight change of $10^{-7}$ as stop criterion resulted in stable decompositions after $<800$ iterations. Each IC can be characterized by a time course (IC activation) and a topography (IC map), the latter being given by the inverse weights. The 30 ICs for each subject were screened for maps resembling the typical frontocentral radial ERN topography and a contribution to the ERP difference between incompatible error and incompatible correct trials, that is, a larger negative deflection at the response interval for erroneous trials. This resulted in identification of one IC for each subject, presumably reflecting the contribution of the neural correlate of performance monitoring to the scalp EEG. Figure S3 (available at www. jneurosci.org as supplemental material) shows the individual IC maps identified along with the average map, after root mean square normalization of individual maps (see Fig. 2a). For each subject, the selected IC was then back-projected to the scalp to reveal unique polarity information and microvolt scaling. Figure S4 (available at www.jneurosci.org as supplemental material) shows the resulting ERPs and ERP images. Compared with the original scalp data (Fig. S2, available at www.jneurosci.org as supplemental material), spatiotemporally overlapping contributions were now absent in the IC ERPs and ERP images.

To model the neural source of the selected ICs, the grand average IC map was submitted to BESA 2000, version 4.2 (MEGIS, Graefeling, Germany). A standardized finite element model (FEM), as provided by BESA, was used. It was created from an averaged head of 24 individual MRIs in Talairach space, and this head was also used for display purposes (Fig. 2c). The BESA FEM provides a realistic approximation of three compartments (brain/CSF, skull, scalp) and was applied with default conductivity parameters. An informed dipole seeding approach was used by placing an equivalent current dipole into the RCZ. The location [Talairach coordinates $(x, y, z)=0,20,30]$ was derived from the secondlevel fMRI result from the same subjects in the same recording session, with the $x$-axis value set to zero.

Time-frequency analysis of single-trial IC activations was performed for data collapsed across three frontocentral channels (FC1, FC2, Cz) by convolving the data with a complex Morlet wavelet $w\left(t, f_{0}\right)$ having a Gaussian shape in the time $\left(\sigma_{t}\right)$ and frequency $\left(\sigma_{f}\right)$ domain around the center frequency $f_{0}$. A constant wavelet is characterized by a constant ratio $Q=\left(f_{0} / \sigma_{f}\right)$. We used nonconstant wavelets with $Q$ increasing linearly from five to eight for frequencies from 3.5 to $35 \mathrm{~Hz}$ (step size, 0.5 $\mathrm{Hz}$ ), which results in an increase in spectral versus temporal resolution with increasing frequency. The $Q$ at $5 \mathrm{~Hz}$ was characterized by a frequency resolution of $\sigma_{f}=0.97 \mathrm{~Hz}$ and a temporal resolution of $\sigma_{t}=165$ $\mathrm{ms}$. For every single trial, the norm of the complex result of the convolution was computed, scaled to decibels $\left(10 \times \log _{10}\right)$, and normalized by subtracting for each frequency the mean value of the -500 to $-200 \mathrm{~ms}$ prestimulus interval from the poststimulus values. The epoch length for the single-trial analysis was set to $3 \mathrm{~s}$ to ensure that the interval of interest $(-500-1500 \mathrm{~ms}$ relative to target onset) did not interfere with invalid edge effects, as indicated by the half length of the wavelet scales.

Based on the results of the time-frequency analysis, which suggested a low $\beta$ increase at about the response interval, the back-projected IC activations were $2-10 \mathrm{~Hz}$ bandpass filtered to unveil a cleaner single-trial estimate of the ERN-related theta response. A parametric vector for each subject was then computed as follows. First, the minimum value in the interval 15-85 ms after each button-press was determined, and the mean of the preceding $(-80-0 \mathrm{~ms})$ and succeeding $(85-240 \mathrm{~ms})$ positivity was subtracted (see Fig. $3 a$ ). These latency windows were determined based on the grand average IC ERP (see Fig. $2 b$ ) and also were compatible with the time-frequency results confirming a prominent theta activity (see Fig. 2e). The resulting single-trial amplitudes vector (see Fig. $3 b$ ) was then convolved with the canonical hemodynamic response function and used as parametric regressor for $\mathrm{fMRI}$ analysis (see below).

MRI data analysis. MR data processing was performed using the software package LIPSIA (Lohmann et al., 2001). Functional data were corrected for slice-time acquisition differences using sinc-interpolation. Signal changes and baseline drifts were removed by applying a temporal high-pass filter with a cutoff frequency of $1 / 120 \mathrm{~Hz}$. Spatial smoothing was applied using a Gaussian filter with $5.65 \mathrm{~mm}$ full width at half maximum (FWHM). To align the functional data slices with a three- dimensional stereotactic coordinate reference system, a rigid linear registration with six degrees of freedom (three rotational and three translational) was performed. The rotational and translational parameters were acquired on the basis of the MDEFT and EPI-T1 slices to achieve an optimal match between these slices and the individual threedimensional reference data set [MDEFT volume data set with 160 slices and $1 \mathrm{~mm}$ slice thickness standardized to the Talairach stereotactic space (Talairach and Tournoux, 1988)] that was acquired for each subject during a previous scanning session. The rotational and translational parameters were subsequently transformed by linear scaling to a standard size. The resulting parameters were then used to transform the functional slices using trilinear interpolation so that the resulting functional slices were aligned with the stereotactic coordinate system, generating output data with a spatial resolution of $3 \mathrm{~mm}^{3}$.

Statistical analysis. Randomization statistics based on 1000 repetitions were performed to identify significant power changes in the time-frequency plane relative to baseline activity in the EEG. This analysis was applied separately to each subject and each condition. In addition, randomization statistics on the time-frequency power differences between incompatible error and incompatible correct conditions were performed. To summarize these results for the group of 13 subjects, binomial statistical analysis was applied.

Statistical analysis of the association between single-trial EEG amplitudes and reaction times was achieved by determining the linear regression slopes between single-trial amplitudes and reaction time values (see Fig. 3c). Separate analyses were performed for the current trial, that is, single-trial amplitudes were related to reaction times of the same trial and to the following trial. In the latter case, single-trial amplitude values were associated to reaction times whenever the following trial belonged to the same stimulus condition. For group analysis, the resulting individual slopes were tested against zero (see Fig. $3 d$ ) by applying one-sided $t$ tests for conditions in which a prediction could be made on the basis of the performance monitoring model.

The statistical analysis of fMRI data was based on a least squares estimation using the general linear model for serially autocorrelated observations (random effects model) (Friston et al., 1995; Worsley and Friston, 1995; Aguirre et al., 1997; Zarahn et al., 1997). Event-related designs were implemented, that is, the hemodynamic response function was modeled by the experimental conditions for each stimulus (event $=$ onset of stimulus presentation). The measured signal was described by a convolution of the temporal stimulus distribution and the hemodynamic response function. The design matrix was generated using a synthetic hemodynamic response function and its first and second derivative and a response delay of $6 \mathrm{~s}$ (Friston et al., 1998). The model equation, including the observation data, the design matrix, and the error term, was convolved with a Gaussian kernel with a dispersion of 4 s FWHM. The effective degrees of freedom were estimated as described by Worsley and Friston (1995). In the following, contrast maps, that is, estimates of the raw-score differences among specified conditions, were generated for each subject. The individual functional datasets were all aligned to the same stereotactic reference space, and a group analysis was performed. For multisession analysis, the random-effects analysis can be effected as a one-sample $t$ test on the resulting contrast images across subjects and sessions (Worsley and Friston, 1995; Holmes and Friston, 1998). Subsequently, $t$ values were transformed into $z$ scores. The design matrix consisted of onset vectors for compatible correct, incompatible correct, and incompatible erroneous trials. Trials involving late response feedbacks and nonevents formed two additional onset vectors. The six translational and rotational motion correction parameters provided by the Siemens motion correction protocol were included as regressors. As in previous studies (Ullsperger and von Cramon, 2001, 2004), analysis of errorrelated brain activity was performed by contrasting incompatible erroneous with incompatible correct trials, thus extracting specific signal increases on errors. Conflict-related activity should cancel out, because response conflict occurs on both incompatible correct and erroneous trials. To minimize the probability of false positives (type I error), only voxels with a $z$ score $>3.09$ ( $p<0.001$, uncorrected $)$ and with a volume $>180 \mathrm{~mm}^{3}$ (five voxels) were considered as activated voxels (Braver et al., 2001). 


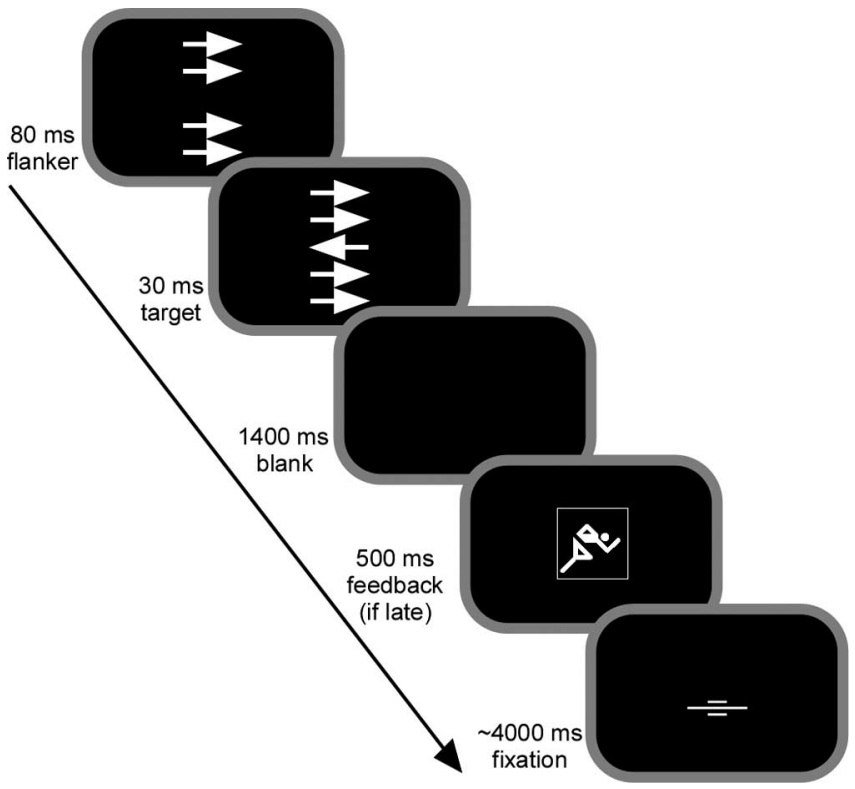

Figure 1. Sequence of stimulus events in the speeded flanker task. Participants viewed four task-irrelevant flanker arrows, followed by a central arrow that indicated the response direction and pointed to the same or opposite direction as flanker arrows. Compatible (same direction) and incompatible (opposite direction) trials appeared in randomized order and with the same probability. For trials in which subjects responded slower than a dynamically adapting individual response deadline, a symbolic feedback occurred urging the subject to speed up in consecutive trials.

In a second analysis testing whether the single-trial ERN measure covaries with the fMRI signal, a parametric design was used (Büchel et al., 1996, 1998). The single-trial amplitudes vector was used as a parameter referring to the onsets of all responses in the task, regardless of their accuracy and speed. The design matrix furthermore contained two onset vectors for late-response feedbacks and nonevents. The six translational and rotational motion correction parameters were included as additional regressors.

\section{Results}

\section{Behavioral results}

While participants underwent concurrent EEG and fMRI data acquisition, they performed a speeded flanker task. They were required to respond with button-presses according to the direction indicated by a centrally presented target arrow, which was surrounded by irrelevant but distracting flanker arrows (Fig. 1). Participants made errors on $0.58 \%$ (SEM, 0.16) of compatible and $17.23 \%$ (SEM, 2.17) of incompatible trials (significant difference; $\left.t_{(12)}=7.75 ; p<0.0001\right)$. The number of compatible errors was insufficient for meaningful statistical analyses, such that this stimulus-response type was excluded from further analysis. Hit reaction times were $380.8 \mathrm{~ms}$ (SEM, 7.9) for compatible and $445.0 \mathrm{~ms}$ (SEM, 8.1) for incompatible trials (significant difference; $\left.t_{(12)}=21.26 ; p<0.0001\right)$. The mean deadline was $475.7 \mathrm{~ms}$ (SEM, 17.7). It was missed in $9.46 \%$ (SEM, 1.8) of compatible and in $27.69 \%$ (SEM, 3.50) of incompatible trials. Error reaction times for incompatible trials were $388.2 \mathrm{~ms}$ (SEM, 9.8), thus being significantly shorter than for incompatible correct trials $\left(t_{(12)}=4.41 ; p<0.001\right)$. In sum, these behavioral results are consistent with previous findings for flanker tasks (Eriksen and Eriksen, 1974; Ullsperger and von Cramon, 2001). Importantly, there was a sufficient numbers of errors for incompatible trials to allow meaningful data analyses. a

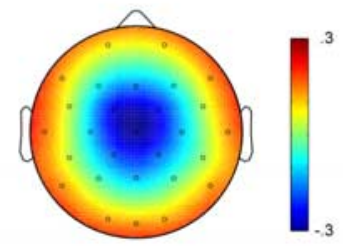

C

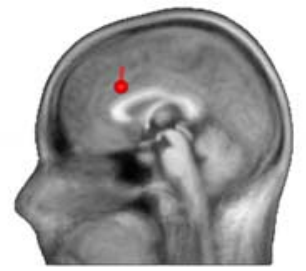

RV $9.8 \%[0,20,30]$

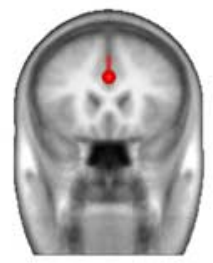

d

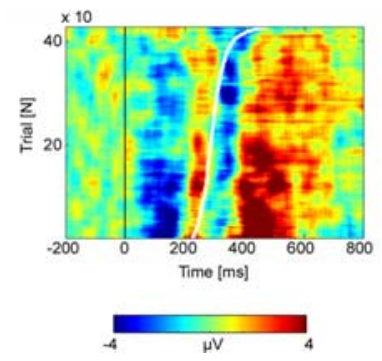

b

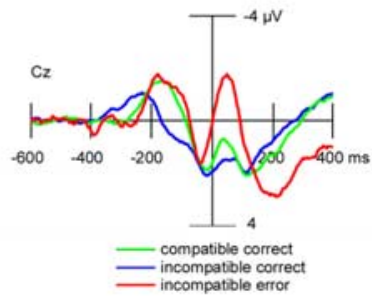

Figure 2. The selected ICs are equivalent to the scalp-recorded ERN. $\boldsymbol{a}$, Identified components were characterized by a radial central topography. Depicted is the grand mean IC topography, after root-mean-square normalization (arbitrary units). $\boldsymbol{b}$, Grand average ( $n=13$ subjects) IC activation ERPs for the vertex electrode (Cz), time-locked to response-onset times, revealed the ERN in the incompatible error condition. Negativity is plotted upwards. $c$, Informed dipole seeding of the grand mean IC topography shown in $\boldsymbol{a}$, at Talairach coordinates $(x, y, z)=$ $0,20,30$. This location was derived from standard analysis of the concurrently recorded fMRI contrasting incompatible error and incompatible correct trials. The equivalent current dipole, which explained $90.2 \%$ of the variance, is plotted on a canonical magnetic resonance image template of the human head. RV, Residual variance. $\boldsymbol{d}$, ERP-image plot of IC incompatible error trials at vertex electrode $(\mathrm{Cz})$ aligned to stimulus onset $(0)$. Sorting the trials by reaction time (sigmoid white line) and smoothing with a moving average across 30 trials visualizes the ERNreaction time relationship. $\boldsymbol{e}$, Time-frequency analysis of the total power difference (in decibels) between IC incompatible error and incompatible correct trials. Significantly more theta activity for the error condition is indicated by the black contour line. The white vertical lines denote the stimulus onset time $(0 \mathrm{~ms})$ and mean reaction time for erroneous responses, respectively.

\section{EEG signal analysis and identification of independent component related to performance monitoring}

After denoising the EEG from MR gradient and ballistocardiac artifacts, ERPs were computed (Fig. S1, available at www.jneurosci. org as supplemental material). As expected, a frontocentral ERN was clearly visible for incompatible error trials but strongly reduced, if not absent, for the correct response conditions (Fig. S2, available at www.jneurosci.org as supplemental material). To separate ERN-related EEG signals from other brain processes and artifacts, we performed ICA on each subject's MR-denoised raw EEG data (Bell and Sejnowski, 1995). ICA returns a set of spatial filters, which yield component activations that are maximally temporally independent from each other. In each subject, we identified one IC that met the following two criteria and therefore was the best candidate to account for the ERN. First, the IC 
should have a near-radial central topography. Second, its backprojected ERP time course should yield a more negative deflection at the response interval in the incompatible error compared with the incompatible correct condition. The topographies of the selected ICs for each subject are shown in the supplemental material (Fig. S3, available at www.jneurosci.org). Independent components reflecting the parietal error positivity $(\mathrm{Pe})$ or the N200 were not observed.

We performed several analyses to test whether the IC selected for each subject indeed reflects the spatial and temporal characteristics of the ERN. First, we performed fMRI-informed source modeling of the grand average IC map (Fig. 2a,c). A single equivalent current dipole was seeded into the RCZ, with the exact location taken from the conventional second-level fMRI analysis contrasting incompatible error and incompatible correct trials [Talairach coordinates $(x, y, z)=0,20,30]$ (Table 1) (see Fig. 5). This source dipole accounted for $90.2 \%$ of the variance (Fig. $2 c$ ). Seeding additional dipoles into other cortical areas identified by the same fMRI analysis (lateral prefrontal cortex, left anterior inferior insula) did not improve the model fit, suggesting that the identified ICs represent activity originating in the RCZ.

Second, grand mean ERPs of the back-projected IC activations revealed a clear ERN in the incompatible error condition (Fig. 2b). These IC ERPs strongly resembled those from the original scalp channel data, but their topography appeared now free of artifact contributions (Fig. S4, available at www.jneurosci.org as supplemental material). Typically, the ERPs showed a polarity reversal at outer electrodes (e.g., F7/8, P7/8) (see Fig. S4, available at www.jneurosci.org as supplemental material), which, taking into account the common average reference, supports the notion that the selected IC can be explained by a single equivalent dipole located in the RCZ.

Third, we explored the EEG signal on a single-trial level. ERPs are usually derived by averaging across trials, which prevents the study of trial-by-trial variations of the EEG signal. To overcome this limitation, so-called ERP images were computed showing the respective single-trial IC activations sorted by reaction time. These analyses demonstrate a good signal-to-noise ratio, because the negativity immediately after erroneous responses was evident in most trials (Fig. 2d).

Fourth, we performed a time-frequency analysis of the IC single-trial signals. ERPs and ERP images both suggest that the ERN was preceded and followed by a positive deflection, indicating an oscillation in the theta frequency range (Luu et al., 2004). Indeed, time-frequency analysis revealed a prominent theta power increase after stimulus onset lasting for $\sim 600 \mathrm{~ms}$. This theta power increase was significant in 11 of 13 subjects for the incompatible correct condition and in 10 of 13 subjects in the incompatible error condition (randomization test; $p<0.01$ ). At the time of the erroneous response, the theta power increase was significantly stronger in incompatible error than in incompatible correct trials (Fig. 2e) (binomial; $p<0.00001$ ). In summary, component map topography, fMRI-informed source modeling, ERP component morphology, and time-frequency results jointly led us to conclude that the IC identified in each subject is very likely equivalent to the ERN as usually obtained outside the MR environment.

\section{Single-trial EEG amplitudes and the dynamics of performance monitoring}

A key prediction that can be derived from performance monitoring models is that physiological signatures of error monitoring should predict behavioral adjustments at the single-trial level. To address this, ERN magnitude was obtained in every single trial by taking peak-to-peak measures of the $2-10 \mathrm{~Hz}$ bandpass-filtered IC signals (Fig. 3a). We examined the relationship of the resulting single-trial amplitudes for each subject (Fig. 3b) to behavior (Fig. $3 c$ ). For incompatible errors, the single-trial amplitude was significantly correlated with reaction time, such that short RTs were associated with high single-trial amplitudes $(b=5.48 ; p=0.002$; one-sided $t$ test). Interestingly, the opposite relationship was found for incompatible correct trials. Here, short RTs were associated with small single-trial amplitudes $(b=-2.14 ; p=0.046)$, consistent with previous findings for the ERN in speeded tasks using a deadline procedure (Luu et al., 2000). However, these effects did not remain significant when RT and single-trial amplitude outliers (values $>3 \mathrm{SD}$ ) and trials followed by a late feedback were excluded from the analysis (incompatible errors, $b=2.61, p=0.103$; incompatible correct, $b=-2.01, p=0.140$ ).

More importantly, we identified a clear relationship between ERN dynamics and subsequent behavioral adjustments. We found that higher single-trial amplitudes were associated with longer RTs on conflict trials after errors (Fig. $3 c, d$ ). Thus, activity variations of the performance monitoring system, as estimated by the single-trial EEG measure, significantly predicted posterror slowing $(b=-5.38 ; p=0.043)$. After exclusion of outliers and trials followed by the late feedback, this effect was even more pronounced $(b=-5.90 ; p=0.032)$. Moreover, this effect could not be explained by a correlation between RTs on errors and RTs on subsequent trials, which was absent (second level mean; $r=$ 0.06; NS). The present analysis thus demonstrates that posterror slowing (Rabbitt, 1966) was driven by postresponse electrophysiological activity in the RCZ.

\section{Trial-by-trial coupling of EEG and fMRI}

A key question in the present context is whether hemodynamic signals related to error monitoring covary with the single-trial ERN (Nunez and Silberstein, 2000; Logothetis et al., 2001). If present, such a relationship should only be found in brain regions specifically involved in performance monitoring. To this end, we used the EEG single-trial amplitude to predict the fMRI BOLD signal. To take into account the slow time course of fMRI BOLD, the single-trial EEG amplitudes were convolved with the hemodynamic response function at button-press onset times. The following parametric random effects fMRI analysis identified a significant correlation of the single-trial amplitude with the BOLD response specifically in the RCZ (Fig. 4, Table 1). The direction of 
a

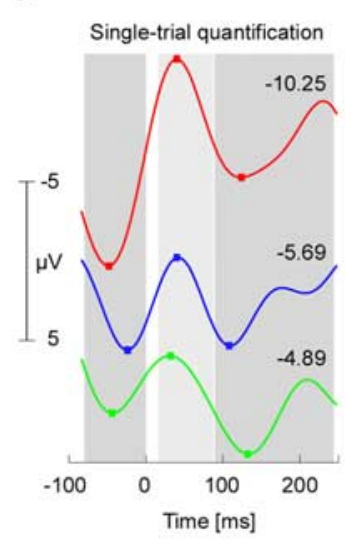

C

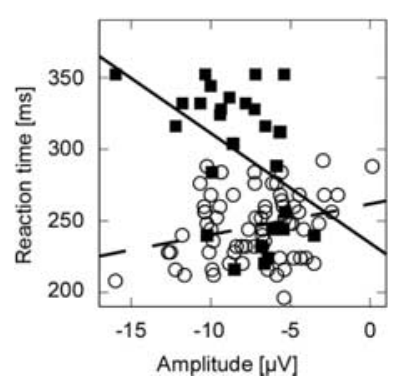

b

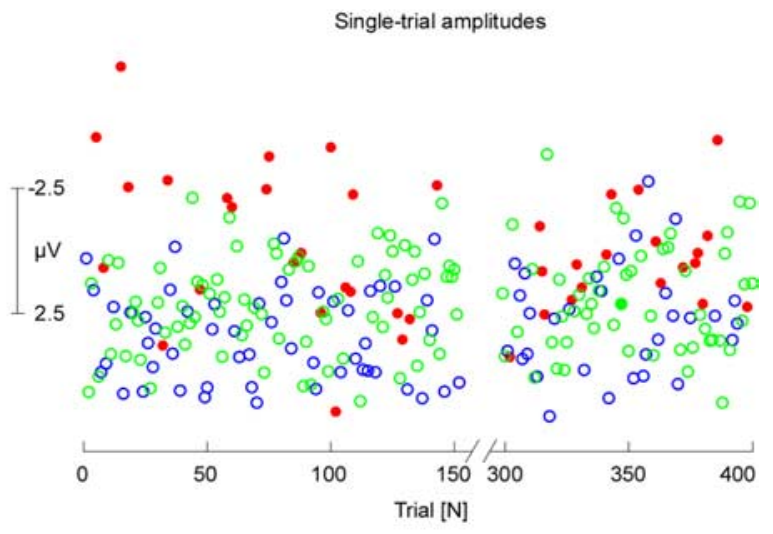

d

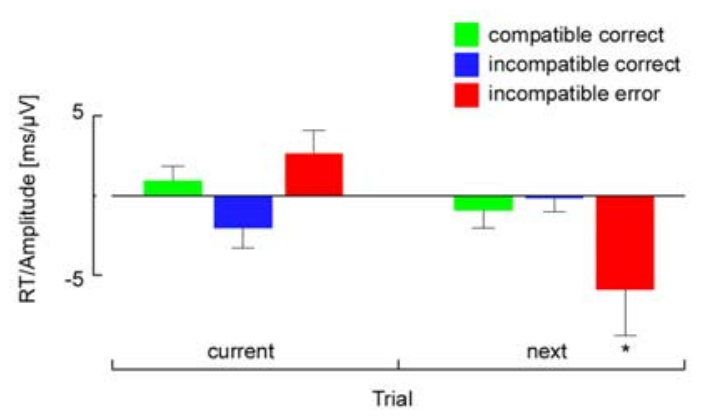

Figure 3. $a$, Quantification of IC single-trial amplitudes exemplified for three trials from the three different conditions. The color code is as in $\boldsymbol{d}$. The mean of the ERN preceding $(-80-0 \mathrm{~ms})$ and after $(85-240 \mathrm{~ms})$ positive peaks was subtracted from the negative peak occurring after each button-press (15-85 ms), giving an amplitude for every single trial. The numbers state the corresponding single-trial amplitude. $\boldsymbol{b}$, Resulting single-trial amplitudes for a representative subject over the course of $\sim 250$ trials (of 400 trials in total). For visualization, the single single-trial amplitude values are color-coded according to the stimulusresponse condition. The color code is as in $\boldsymbol{d}$. Note the considerable amount of variance within each experimental condition presumably reflecting the varying strength of performance monitoring. c, Single-subject example showing for the incompatible error condition the relationship between single-trial amplitude and reaction time, separately for the current trial (open circles; dashed regression curve) and for the reaction time of the following trial (filled squares; solid regression curve). $\boldsymbol{d}$, Second-level result across all $n=13$ subjects, showing the mean reaction time to single-trial amplitudes slope ( + SEM) for all three conditions after removal of outlier ( $>3$ SD) and late feedback trials, separately for the same trial and for the next trial. ${ }^{*} p<0.05$.

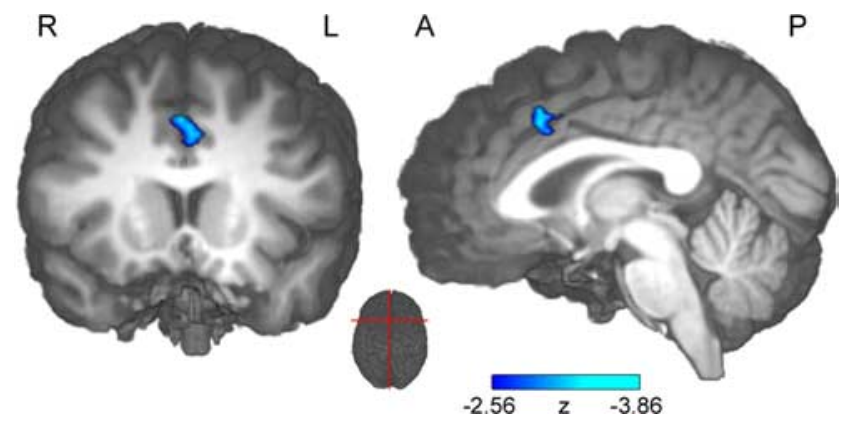

Figure 4. Result of the EEG-informed parametric fMRI analysis based on IC single-trial amplitudes, plotted on an individual brain. FMRI signals correlated with single-trial amplitudes solely in the RCZ along the banks of the cingulate sulcus [center of gravity at coordinates $(x, y$, $z)=0,17,42 ; z=-3.86]$. The left part shows coronal view; the right part shows the sagittal view on the right hemisphere. The red lines on the middle top view inset indicate slice sections. $R$, Right; L, left; A, anterior; P, posterior.

the observed relationship confirmed that trials with greater absolute single-trial ERN amplitudes were associated with stronger BOLD responses in the RCZ. Although the EEG-informed approach as well as the conventional fMRI analysis identified the
RCZ, the conventional fMRI contrast revealed additional brain structures, such as insular and lateral prefrontal cortex (Fig. 5 , Table 1). The conventional contrast extracted error-specific signal increases, whereas performance-monitoring-related activations present in both correct and incorrect trials cancelled out to a large degree. The EEG-informed fMRI analysis, on the other hand, was more specific to variations of the monitoring signal itself. These differences in process specificity might also account for the observation that the maxima of the RCZ foci were separated by $\sim 12 \mathrm{~mm}$ in inferior-superior direction (Ullsperger and von Cramon, 2001). However, with the given the between-plane resolution, a functional interpretation of this finding remains speculative.

\section{Discussion}

The present study demonstrates an eventrelated trial-by-trial coupling of simultaneously recorded EEG, fMRI, and behavior in humans. The major advantage of simultaneous recordings is that these different measures are studied under identical sensory and motivational conditions, thereby allowing the investigation of trialby-trial fluctuations. We found the singletrial ERN to be systematically related to ensuing behavioral adjustments. As predicted by performance-monitoring models, the ERN seems to signify the likelihood that the action outcome is worse than expected (Holroyd et al., 2004; Brown and Braver, 2005). On error trials, this likelihood estimate has been suggested to be driven primarily by postresponse conflict between executed and concurrently activated response tendencies (Yeung et al., 2004). Moreover, the single-trial ERN amplitude reflects activation of the RCZ neurons involved in controlling subsequent adjustments. This finding is paralleled by invasive recordings showing that performance adjustments are preceded by enhanced firing rates of RCZ neurons (Shima and Tanji, 1998; Williams et al., 2004). Our finding that posterror slowing was predicted by the single-trial ERN has not been reported in previous studies, in which this relationship was investigated by the study of the averaged ERN and correlations across subjects. At the group level, however, many other factors may contribute to averaged ERN amplitude variations (e.g., skull thickness, variations of the cingulate sulcus, and trait factors) (Pailing and Segalowitz, 2004). Therefore, a weak relationship between ERN and posterror slowing can be easily missed because of lack of statistical power, which might help to explain previously published mixed findings on the posterror slowing effect. In line with our results, however, is one study investigating this relationship at a within-subjects level and reporting that larger ERN amplitudes predicted increased posterror slowing (Gehring et al., 1993). We conclude that the singletrial measure of the ERN reflects trial-by-trial fluctuations in the activity of performance monitoring circuits that are responsible 
for behavioral adjustments. The ability to measure the dynamics of the monitoring signal will significantly facilitate addressing its relationship to immediate and longterm control adjustments (Ridderinkhof et al., 2004).

The peak-to-peak measure used to quantify performance-monitoring-related EEG activity takes into account the ERN and the subsequent positive peak, which is also maximal at frontocentral sites. This frontocentral positive EEG deflection seems to result from activity in the RCZ as well, which is consistent with a previous source localization study (van Veen and Carter, 2002). Note that this positivity should not be confused with the parietal error positivity Pe (Falkenstein et al., 1990, 2000), which was not captured in the ICA decomposition. Furthermore, it has been hypothesized that ERN and preresponse N200 on correct trials may both reflect similar processes, namely response conflict monitoring (Yeung et al., 2004). We did not obtain a reliable N200 conflict effect in our data; in particular, we found no IC reflecting an N200 modulation. Usually, the N200 preresponse conflict effect is very small (up to $2 \mu \mathrm{V}$ ) (van Veen and Carter, 2002), and we therefore may have missed it as a result of the recording environment or the limited number of electrodes. The relationship between ERN and the N200 conflict effect remains an open question that should be optimally addressed by ICA decomposition of high-density EEG data acquired outside the MR environment.

For resting conditions, it has been shown previously that simultaneous EEG and fMRI BOLD can reveal systematic correlations (Goldman et al., 2002; Laufs et al., 2003). The event-related trial-by-trial correlation approach applied here presents a new strategy for integrating EEG and fMRI. Alternative approaches such as fMRI-informed ERP dipole seeding (Ullsperger and von Cramon, 2001; Thees et al., 2003) implicitly assume a tight link between the neural generators of ERPs and fMRI activation foci. However, as exemplified by the present study, conventional fMRI contrasts may well reveal cortical regions that do not comprise neuronal sources of the ERP and vice versa (Nunez and Silberstein, 2000). Because fMRI BOLD and ERP components can differ with regard to their sensitivity to experimental manipulations, fMRI-informed dipole seeding is not necessarily a valid solution of the inverse problem in EEG.

High magnetic fields provide adverse conditions for EEG recordings. Application of ICA offers a practical solution to minimize artifacts and to identify functionally meaningful EEG activity on a trial-by-trial basis (Debener et al., 2005; Makeig et al., 2002, 2004). The identified IC reflected all features of the ERN, including scalp topography, ERP morphology, and time-frequency characteristics. We also found that the fMRI-informed dipole seeding approach confirmed the RCZ as the major source of the selected ICA correlate of the ERN. This latter finding also validates the main assumption of ICA as applied to EEG data. Under favorable circumstances, a clean independent component should reflect the ongoing activity of a synchronous piece of cortex expressing a dipolar projection. More importantly, we conclude that the covariation of the single-trial ERN measure with the fMRI BOLD response in the RCZ strongly supports the proposed RCZ source of the ERN (Holroyd et al., 2004; Ridderinkhof et al., 2004; Ullsperger and von Cramon, 2004). Thus, the fMRI signal and the ICA single-trial correlate of the ERN appear to reflect directly related neuronal and metabolic processes in the RCZ. Our data fit well with animal research on a coupling between BOLD and local field potentials (Logothetis et al., 2001; Logothetis, 2003), the latter being known to be the basis of the scalp-recorded EEG (Nunez and Silberstein, 2000).

The joint study of electrophysiological and hemodynamic signatures of regional event-related brain activity seems very promising because future studies may succeed in identifying multiple functionally relevant ICs. This would allow to relate several IC time courses to regional fMRI activation, thereby holding great potential to address two cardinal questions in the field of cognitive neuroscience. First, concurrent single-trial EEG/fMRI will facilitate addressing the dynamic interplay between ongoing and event-related brain activity (Arieli et al., 1996; Makeig et al., 2002). Second, adding electrophysiological information provides high temporal precision and thus helps to shape models on how cognitive processes are dynamically implemented in distinct cortical areas (Stephan et al., 2004). By successfully demonstrating a trial-by-trial coupling of noninvasive event-related EEG and fMRI, new avenues are opened for future experiments that address the dynamics of information processing within both anatomically and functionally defined neural networks.

\section{References}

Aguirre GK, Zarahn E, D’Esposito M (1997) Empirical analyses of BOLD fMRI statistics. II. Spatially smoothed data collected under nullhypothesis and experimental conditions. NeuroImage 5:199-212.

Allen PJ, Polizzi G, Krakow K, Fish DR, Lemieux L (1998) Identification of EEG events in the MR scanner: the problem of pulse artifact and a method for its subtraction. NeuroImage 8:229-239.

Allen PJ, Josephs O, Turner R (2000) A method for removing imaging artifact from continuous EEG recorded during functional MRI. NeuroImage $12: 230-239$.

Arieli A, Sterkin A, Grinvald A, Aertsen A (1996) Dynamics of ongoing 
activity: explanation of the large variability in evoked cortical responses. Science 273:1868-1871.

Bell AJ, Sejnowski TJ (1995) An information-maximization approach to blind separation and blind deconvolution. Neural Comput 7:1129-1159.

Braver TS, Barch DM, Kelley WM, Buckner RL, Cohen NJ, Miezin FM, Snyder AZ, Ollinger JM, Akbudak E, Conturo TE, Petersen SE (2001) Direct comparison of prefrontal cortex regions engaged by working and longterm memory tasks. NeuroImage 14:48-59.

Brown JW, Braver TS (2005) Learned predictions of error likelihood in the anterior cingulate cortex. Science 307:1118-1121.

Büchel C, Wise RJS, Mummery CJ, Poline J-B, Friston KJ (1996) Nonlinear regression in parametric activation studies. NeuroImage 4:60-66.

Büchel C, Holmes AP, Rees G, Friston KJ (1998) Characterizing stimulusresponse functions using nonlinear regressors in parametric fMRI experiments. NeuroImage 8:140-148.

Debener S, Makeig S, Delorme A, Engel AK (2005) What is novel in the novelty oddball paradigm? Functional significance of the novelty P3 event-related potential as revealed by independent component analysis. Brain Res Cogn Brain Res 22:309-321.

Dehaene S, Posner MI, Tucker DM (1994) Localization of a neural system for error detection and compensation. Psychol Sci 5:303-305.

Delorme A, Makeig S (2004) EEGLAB: an open source toolbox for analysis of single trial EEG dynamics including independent component analysis. J Neurosci Methods 134:9-21.

Eriksen BA, Eriksen CW (1974) Effects of noise letters upon the identification of a target letter in a nonsearch task. Percept Psychophys 16:143-149.

Falkenstein M, Hohnsbein J, Hoormann J, Blanke L (1990) Effects of errors in choice reaction tasks on the ERP under focused and divided attention. In: Psychophysiological brain research (Brunia CHM, Gaillard AWK, Kok A, eds), pp 192-195. Tilburg, The Netherlands: Tilburg UP.

Falkenstein M, Hoormann J, Christ S, Hohnsbein J (2000) ERP components on reaction errors and their functional significance: a tutorial. Biol Psychol 51:87-107.

Friston KJ, Holmes AP, Poline JB, Grasby PJ, Williams SC, Frackowiak RS, Turner R (1995) Analysis of fMRI time-series revisited. NeuroImage 2:45-53.

Friston KJ, Fletcher P, Josephs O, Holmes A, Rugg MD, Turner R (1998) Event-related fMRI: characterizing differential responses. NeuroImage 7:30-40.

Garavan H, Ross TJ, Murphy K, Roche RA, Stein EA (2002) Dissociable executive functions in the dynamic control of behavior: inhibition, error detection, and correction. NeuroImage 17:1820-1829.

Gehring WJ, Goss B, Coles MG, Meyer DE, Donchin E (1993) A neural system for error detection and compensation. Psychol Sci 4:385-390.

Goldman RI, Stern JM, Engel Jr J, Cohen MS (2002) Simultaneous EEG and fMRI of the alpha rhythm. NeuroReport 13:2487-2492.

Holmes AP, Friston KJ (1998) Generalisability, random effects and population inference. NeuroImage 7:754.

Holroyd CB, Nieuwenhuis S, Mars RB, Coles MGH (2004) Anterior cingulate cortex, selection for action, and error processing. In: Cognitive neuroscience of attention (Posner MI, ed), pp 219-231. New York: The Guilford Press.

Josephs O, Turner R, Friston K (1997) Event-related fMRI. Hum Brain Mapp 5:243-248.

Kerns JG, Cohen JD, MacDonald III AW, Cho RY, Stenger VA, Carter CS (2004) Anterior cingulate conflict monitoring and adjustments in control. Science 303:1023-1026.

Laufs H, Krakow K, Sterzer P, Eger E, Beyerle A, Salek-Haddadi A, Kleinschmidt A (2003) Electroencephalographic signatures of attentional and cognitive default modes in spontaneous brain activity fluctuations at rest. Proc Natl Acad Sci USA 100:11053-11058.

Lee TW, Girolami M, Sejnowski TJ (1999) Independent component analysis using an extended infomax algorithm for mixed subgaussian and supergaussian sources. Neural Comput 11:417-441.
Logothetis NK (2003) The underpinnings of the BOLD functional magnetic resonance imaging signal. J Neurosci 23:3963-3971.

Logothetis NK, Pauls J, Augath M, Trinath T, Oeltermann A (2001) Neurophysiological investigation of the basis of the fMRI signal. Nature 412:150-157.

Lohmann G, Müller K, Bosch V, Mentzel H, Hessler S, Chen L (2001) LIPSIA-a new software system for the evaluation of functional magnetic resonance images of the human brain. Comput Med Imaging Graph 25:449-457.

Luu P, Flaisch T, Tucker DM (2000) Medial frontal cortex in action monitoring. J Neurosci 20:464-469.

Luu P, Tucker DM, Makeig S (2004) Frontal midline theta and the errorrelated negativity: neurophysiological mechanisms of action regulation. Clin Neurophysiol 115:1821-1835.

Makeig S, Westerfield M, Jung TP, Enghoff S, Townsend J, Courchesne E, Sejnowski TJ (2002) Dynamic brain sources of visual evoked responses. Science 295:690-694.

Makeig S, Debener S, Onton J, Delorme A (2004) Mining event-related brain dynamics. Trends Cogn Sci 8:204-210.

Miezin FM, Maccotta L, Ollinger JM, Petersen SE, Buckner RL (2000) Characterizing the hemodynamic response: effects of presentation rate, sampling procedure, and the possibility of ordering brain activity based on relative timing. NeuroImage 11:735-759.

Niazy RK, Beckmann CF, Iannetti GD, Brady JM, Smith SM (2005) Removal of FMRI environment artifacts from EEG data using optimal basis sets. NeuroImage 28:720-737.

Nunez PL, Silberstein RB (2000) On the relationship of synaptic activity to macroscopic measurements: does co-registration of EEG with fMRI make sense? Brain Topogr 13:79-96.

Pailing PE, Segalowitz SJ (2004) The error-related negativity as a state and trait measure: motivation, personality, and ERPs in response to errors. Psychophysiology 41:84-95.

Rabbitt PM (1966) Errors and error correction in choice-response tasks. J Exp Psychol 71:264-272.

Ridderinkhof KR, Ullsperger M, Crone EA, Nieuwenhuis S (2004) The role of the medial frontal cortex in cognitive control. Science 306:443-447.

Shima K, Tanji J (1998) Role for cingulate motor area cells in voluntary movement selection based on reward. Science 282:1335-1338.

Stephan KE, Harrison LM, Penny WD, Friston KJ (2004) Biophysical models of fMRI responses. Curr Opin Neurobiol 14:629-635.

Talairach P, Tournoux J (1988) A stereotactic coplanar atlas of the human brain. Stuttgart, Germany: Thieme.

Thees S, Blankenburg F, Taskin B, Curio G, Villringer A (2003) Dipole source localization and fMRI of simultaneously recorded data applied to somatosensory categorization. NeuroImage 18:707-719.

Ullsperger M, von Cramon DY (2001) Subprocesses of performance monitoring: a dissociation of error processing and response competition revealed by event-related fMRI and ERPs. NeuroImage 14:1387-1401.

Ullsperger M, von Cramon DY (2004) Neuroimaging of performance monitoring: error detection and beyond. Cortex 40:593-604.

van Veen V, Carter CS (2002) The timing of action-monitoring processes in the anterior cingulate cortex. J Cogn Neurosci 14:593-602.

Williams ZM, Bush G, Rauch SL, Cosgrove GR, Eskandar EN (2004) Human anterior cingulate neurons and the integration of monetary reward with motor responses. Nat Neurosci 7:1370-1375.

Worsley KJ, Friston KJ (1995) Analysis of fMRI time-series revisited-again. NeuroImage 2:173-181.

Yeung N, Cohen JD, Botvinick MM (2004) The neural basis of error detection: conflict monitoring and the error-related negativity. Psychol Rev 111:931-959.

Zarahn E, Aguirre GK, D’Esposito M (1997) Empirical analyses of BOLD fMRI statistics. I. Spatially unsmoothed data collected under nullhypothesis conditions. NeuroImage 5:179-197. 